\title{
Electrophysiological Characteristics and Radiofrequency Catheter Ablation Treatment of Idiopathic Ventricular Arrhythmias Successfully Ablated From the Ostium of the Coronary Sinus
}

\author{
Yoshiaki Yui, MD; Yukio Sekiguchi, MD; Akihiko Nogami, MD; Hiro Yamasaki, MD; \\ Takeshi Machino, MD; Kenji Kuroki, MD; Miyako Igarashi, MD; Kazutaka Aonuma, MD
}

\begin{abstract}
Background: Idiopathic ventricular arrhythmias (VAs) rarely arise from the epicardium at the crux of the heart. However, the electrophysiological characteristics of VAs successfully ablated from the ostium of the coronary sinus (CSO) have not yet been documented.

Methods and Results: Electrocardiographic and electrophysiological data were analyzed in patients with idiopathic VAs successfully ablated from the CSO. Among 309 patients with idiopathic VAs treated with radiofrequency catheter ablation (RFCA), 6 (1.94\%; 3 men; age: $66.3 \pm 9.7$ years) had VAs successfully ablated from the CSO. Only 1 patient had sustained ventricular tachycardia. The morphology of the QRS showed a left superior axis and QS pattern in leads III and aVF. Furthermore, the precordial maximum deflection index was $>0.55$ in all patients and a right bundle branch block pattern was recorded in 5 of 6 patients. All VAs were successfully eliminated by RFCA within the CSO. Intracardiac ECGs at sites where VAs were eliminated by RFCA showed clear atrial and ventricular potentials (atrial amplitude: $0.21 \pm 0.11 \mathrm{mV}$; ventricular amplitude: $0.43 \pm 0.24 \mathrm{mV}$ ), except in 1 case of atrial fibrillation. No patients had recurrence during the 3.4 \pm 1 .8-year follow-up period.
\end{abstract}

Conclusions: The idiopathic VAs in our study were eliminated by RFCA within the CS, where a clear atrial amplitude was recorded.

Key Words: Catheter ablation; Coronary sinus; Electrophysiology; Ventricular arrhythmias

I diopathic ventricular arrhythmias (VAs) mostly originate from the ventricular endocardium. The arrhythmogenic sites vary, but include the left and right ventricular (RV) outflow tracts, the Purkinje system, ${ }^{1,2}$ mitral ${ }^{3}$ and tricuspid annuli, ${ }^{4}$ and papillary muscles. ${ }^{5,6}$ However, near the left ventricular outflow tract, VAs sometimes originate beside the epicaidium. ${ }^{7,8}$

Identifying the arrhythmogenic foci prior to the ablation procedure is useful and facilitates ablation strategy planning. Daniels et al reported that a maximum deflection index $(\mathrm{MDI})>0.55$ could predict a VA epicardial origin in the left ventricle (LV) with a sensitivity of $100 \%$ and specificity of $98.7 \% .{ }^{9}$ Berruezo et al examined the ECG characteristics of epicardial VAs and determined that pseudo-delta wave duration, intrinsicoid deflection time, and shortened RS complex were predictive of an epicardial origin with a sensitivity of $76-87 \%$ and a specificity of $85-95 \% .^{10}$

In previous reports, catheter ablation has been performed from the inside of the coronary sinus $(\mathrm{CS})^{11-14}$ (i.e., from the epicardial side of the myocardium). In addition, Doppalapudi et al reported 4 cases of idiopathic focal ventricular tachy- cardia (VT) originating from the crux of the heart, located near the junction of the middle cardiac vein (MCV) and the CS. ${ }^{15}$ In that report, successful radiofrequency catheter ablation (RFCA) was performed within the proximal MCV in only 1 patient and another 2 patients were treated with a percutaneous epicardial approach for the VAs. ${ }^{15}$ VAs successfully ablated via the MCV are less frequent than those treated from the great cardiac vein or anterior interventricular vein, ${ }^{16,17}$ and VAs treated from the ostium of the CS (CSO) have also been reported in small studies.9,18 Thus, reports of the electrophysiological characteristics of VAs successfully ablated from the CSO are scarce. As shown in previous reports, ${ }^{11-18}$ some cases of idiopathic VAs have been successfully treated using RFCA from the venous circulation. However, VAs do not have their origins in venous structures, as the venous circulation does not have ventricular myocardium. However, such VAs may arise from ventricular myocardium in close proximity to the venous circulation system. The present study sought to clarify the electrophysiological characteristics of VAs by mapping and ablating them from the epicardium thorough the venous structure.

Received December 12, 2016; revised manuscript received May 8, 2017; accepted May 25, 2017; released online June 23, 2017 Time for primary review: 52 days

Cardiovascular Division, Faculty of Medicine, University of Tsukuba, Tsukuba, Japan

Mailing address: Yukio Sekiguchi, MD, Cardiovascular Division, Faculty of Medicine, University of Tsukuba, 1-1-1 Tennodai, Tsukuba 305-8575, Japan. E-mail: yseki@md.tsukuba.ac.jp

ISSN-1346-9843 All rights are reserved to the Japanese Circulation Society. For permissions, please e-mail: cj@j-circ.or.jp 
A

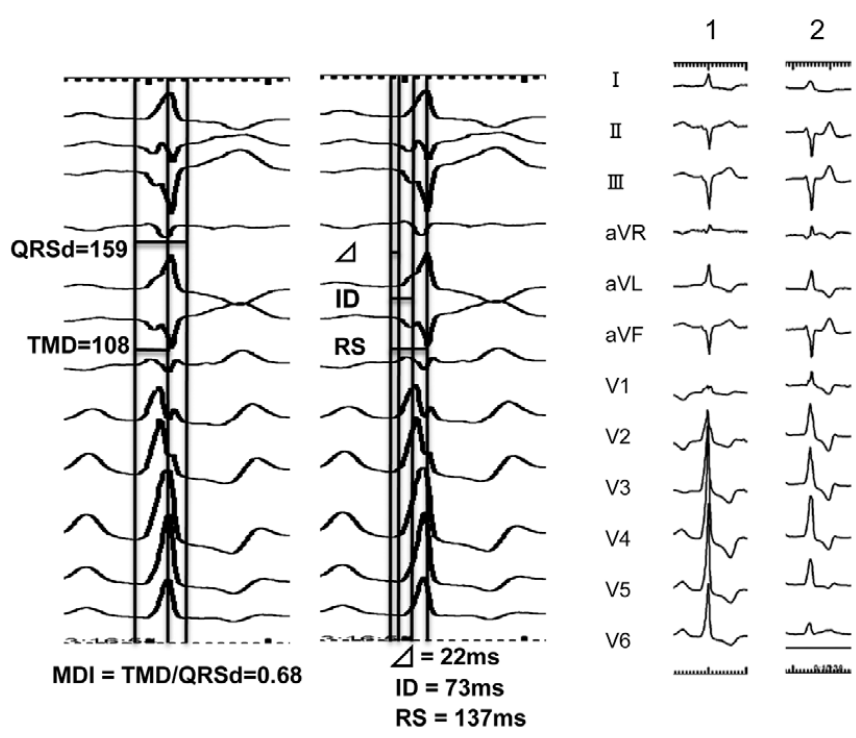

B

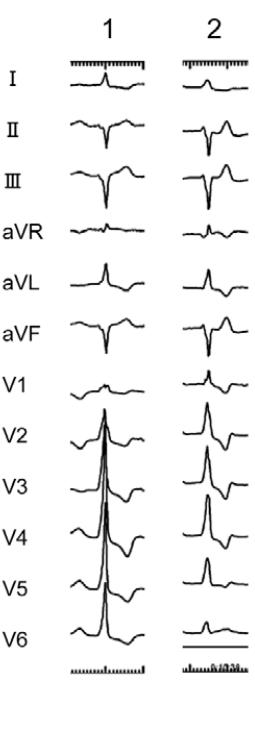

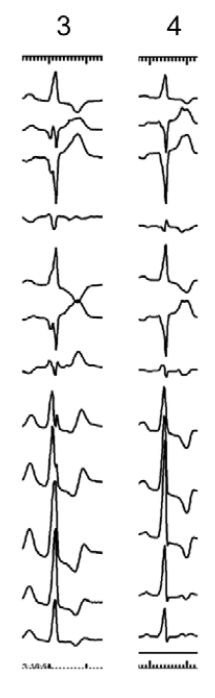

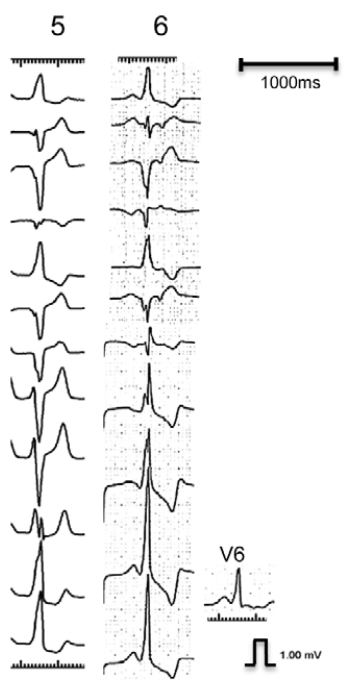

Figure 1. (A) An example of an ECG in patient 3. The maximum deflection index (MDI) was calculated as the earliest time to maximum deflection in any of the precordial leads divided by the total QRS duration. The pseudo-delta $(\Delta)$ wave was measured from the earliest ventricular activation to the earliest fast deflection in any precordial lead. The intrinsicoid deflection time was measured from the earliest ventricular activation to the peak of the R wave in V2. The shortest RS complex (RS) was measured from the earliest ventricular activation to the nadir of the first $S$ wave in any precordial lead. (B) QRS morphology of ventricular arrhythmias (VAs) from the coronary sinus ostium (CSO) in 6 patients. All VAs show a superior axis and positive polarity in leads I and aVL. Right bundle branch block and left bundle branch block are observed in the precordial leads.

\section{Methods}

\section{Definition of the CSO}

We defined the $\mathrm{CSO}$ as the area within $2 \mathrm{~cm}$ of the proximal portion of the main CS trunk and the entrance of the $\mathrm{MCV}$, where it might be easy to manipulate a catheter. The precise position of the CSO was evaluated by CS venography. The distance of $2 \mathrm{~cm}$ was measured using a 3D mapping system (CARTO, Biosense Webster, Diamond Bar, CA, USA; or Ensite Navx St. Jude Medical, Minneapolis, MN, USA).

\section{Study Population}

A total of 309 patients with idiopathic VAs treated with catheter ablation between January 2009 and June 2014 were retrospectively enrolled in this study. All VAs were resistant to medical therapy, and cases of structural heart disease were excluded based on echocardiography, cardiac magnetic resonance imaging, or coronary angiography findings. There were $6(1.94 \%)$ patients treated with RFCA at the CSO. We examined the clinical and electrophysiological characteristics and RFCA outcomes of these patients. Survey items included the patients' characteristics, ECG characteristics, and electrophysiological findings. Ethical approval was given by the local review committee and all patients gave written informed consent.

\section{Analysis of 12-Lead ECGs}

We analyzed the QRS morphology of the sinus rhythm beat, the VAs, the QRS duration of VAs, and the premature ventricular contraction-QRS coupling interval. Moreover, the previously reported ECG characteristics of epicardial
VAs were measured, including MDI, ${ }^{9}$ pseudo-delta wave, and intrinsicoid deflection time, and the shortest RS complex was determined.10 The MDI was calculated as the earliest time to maximum deflection in any of the precordial leads divided by the total QRS duration. The pseudo-delta wave was measured from the earliest ventricular activation to the earliest fast deflection in any precordial lead. The intrinsicoid deflection time was measured from the earliest ventricular activation to the peak of the $\mathrm{R}$ wave in $\mathrm{V} 2$. The shortest RS complex was measured from the earliest ventricular activation to the nadir of the first $\mathrm{S}$ wave in any precordial lead. Each parameter is illustrated in Figure 1A.

\section{Electrophysiological Study of Mapping and Ablation}

Before the procedure, all antiarrhythmic drugs were discontinued for at least 5 half-lives. Patients remained in a fasting state during the electrophysiological study and were sedated with pentazocine and hydroxyzine hydrochloride to relieve any pain. Surface ECGs and intracardiac electrograms from the catheters were continuously monitored and stored on a computer-based digital recording system (Prucka Cardiolab; GE Healthcare Inc., Houston, TX, USA; Bard lab; Bard Inc., Delran, NJ, USA). After the catheters were placed in the His bundle region and RV apex under fluoroscopic guidance via the right femoral vein, we attempted to induce clinical VAs. If VAs did not spontaneously occur, intravenous injection of isoproterenol, programmed ventricular stimulation, and incremental burst pacing were performed from the catheter located at the RV apex. Mapping and ablation were performed using a 7Fr quadripolar nonirrigation catheter with a 4-mm tip (Cordis Webster Inc., Baldwin Park, CA, USA; EP Technologies Inc., San Jose, 
Table 1. Clinical and Arrhythmia Characteristics of the 6 Study Patients

\begin{tabular}{|c|c|c|c|c|c|c|c|}
\hline \multirow[b]{3}{*}{ Clinical } & \multicolumn{6}{|c|}{ Patient no. } & \multirow{2}{*}{ Mean \pm SD } \\
\hline & 1 & 2 & 3 & 4 & 5 & 6 & \\
\hline & & & & & & & \\
\hline Age (years) & 77 & 77 & 71 & 58 & 58 & 57 & $66.3 \pm 9.7$ \\
\hline Sex & Male & Male & Female & Female & Male & Female & \\
\hline LVEF (\%) & 68 & 64 & 50 & 63 & 55 & 63 & $60.5 \pm 6.6$ \\
\hline Rhythm & Sinus & AF & Sinus & Sinus & Sinus & Sinus & \\
\hline Arrhythmia pattern & PVC & NSVT & PVC & NSVT & SVT & PVC & \\
\hline Symptoms & Fatigue & Palpitation & None & Palpitation & Palpitation & Palpitation & \\
\hline Duration of symptoms (years) & 3 & 5 & 1.5 & 1 & 3 & 1 & \\
\hline VE beats (no./24h) & $7,061(9 \%)$ & $6,191(6 \%)$ & $43,728(39 \%)$ & - & $30,112(30 \%)$ & $7,688(8 \%)$ & \\
\hline Successful ablation site & MCV-os & PCS & CSO & MCV-os & CSO & PCS & \\
\hline QRS morphology & LSA & LSA & LSA & LSA & LSA & LSA & \\
\hline QRS duration (ms) & 169 & 134 & 159 & 130 & 170 & 148 & $151.6 \pm 17.2$ \\
\hline PVC coupling interval (ms) & 489 & 479 & 455 & 409 & 494 & 596 & $487 \pm 61.8$ \\
\hline MDI & 0.61 & 0.56 & 0.68 & 0.59 & 0.7 & 0.59 & $0.62 \pm 0.05$ \\
\hline \multicolumn{8}{|l|}{ Berruezo criteria } \\
\hline Pseudo-delta wave (ms) & 32 & 17 & 22 & 27 & 22 & 20 & $23.2 \pm 5.3$ \\
\hline Intrinsicoid deflection (ms) & 88 & 78 & 73 & 79 & 48 & 74 & $73.3 \pm 13.5$ \\
\hline Shortest RS complex (ms) & 141 & 123 & 137 & 115 & 160 & 106 & $130.3 \pm 19.5$ \\
\hline
\end{tabular}

$\mathrm{AF}$, atrial fibrillation; CSO, coronary sinus ostium; LVEF, left ventricular ejection fraction; MCV-os, middle cardiac vein ostium; MDI, maximum deflection index; NSVT, non-sustained ventricular tachycardia; PCS, proximal coronary sinus; PVC, premature ventricular contraction; SVT, sustained ventricular tachycardia; VE, ventricular ectopic.

CA, USA). The origin of the VAs was estimated from the precedence of local activation and pace mapping. In this study, the pace maps were classified into 3 groups: good pace maps: 10/12 matching leads; excellent pace maps: 11/12 matching leads; and perfect pace maps: $12 / 12$ matching leads. First, the VAs were mapped from the RV side in all patients. If the findings of local activation and the pace map from the RV side were good, but not excellent, or not good, mapping from inside the CS was performed. In addition, when the findings of local activation and the pace map from inside the CS were good, but not excellent, mapping from the LV side was performed. The catheter was advanced to the LV by a transaortic or trans-septal approach. The site of VA origin was defined as where the earliest ventricular activation was recorded and/or a perfect pace map could be obtained. RF energy was delivered using a maximum power of $35-50 \mathrm{~W}$ and a maximum temperature of $55^{\circ} \mathrm{C}$ for each delivery. When adequate RF energy could not be obtained because of high temperature of the catheter tip, the 4-mm non-irrigated tip ablation catheter was changed to a $3.5-\mathrm{mm}$ irrigated tip catheter. In addition, when the origin of the target VA was predicted to be at the CSO, an irrigation catheter was used from the beginning of the procedure. Irrigated tip catheter RF applications were performed with a maximum temperature of $43^{\circ} \mathrm{C}$ for each delivery. The precise ablation sites near the CSO were identified by either antegrade or retrograde CS venography. The distance between each ablation site and coronary artery was evaluated by coronary angiography just before RF application. Activation mapping was performed with the CARTO or Ensite Navx electroanatomical mapping system. In this study, the RFCA endpoint was established as complete elimination of the VA, even following pharmaceutical or electrical ventricular stimulation.

\section{Follow-up}

Patients were monitored as outpatients. VA recurrence was evaluated by a 12-lead ECG, 24-h Holter monitoring, event recorder, and patient reports of symptoms for more than 6 months after discharge.

\section{Statistical Analysis}

Continuous variables are expressed as mean \pm standard deviation, and skewed variables are presented as median with interquartile range. As appropriate, the chi-square test or Fisher's exact probability test was used for comparison of categorical variables. To identify differences in 1 patient between 2 time points, the Wilcoxon signed-rank test was used. Differences are reported as significant if the $\mathrm{P}$-value was $<0.05$. All statistical analyses were performed using SPSS software (version 16.0; SPSS Inc., Chicago, IL, USA).

\section{Results}

\section{Patients' Characteristics}

Clinical characteristics of the 6 patients $(66.3 \pm 9.7$ years; range: $57-77$ years; 3 men) who were treated with RFCA at the CSO are shown in Table 1: 5 patients had normal sinus rhythm, and 1 patient had persistent atrial fibrillation for 5 years. Only 1 patient had sustained VT and required an implantable cardioverter defibrillator. Symptoms varied and included palpitation, fatigue, and chest pain. The duration of symptoms was $1-5$ years.

\section{2-Lead ECG}

The 12-lead ECGs are shown in Figure 1B. In all patients, the QRS configuration of clinical VAs was a left superior axis and QS pattern in leads III and aVF. A 'qrS' pattern was recognized in lead II in 2 patients; 5 patients had a right bundle branch block pattern, and 1 had a left bundle 


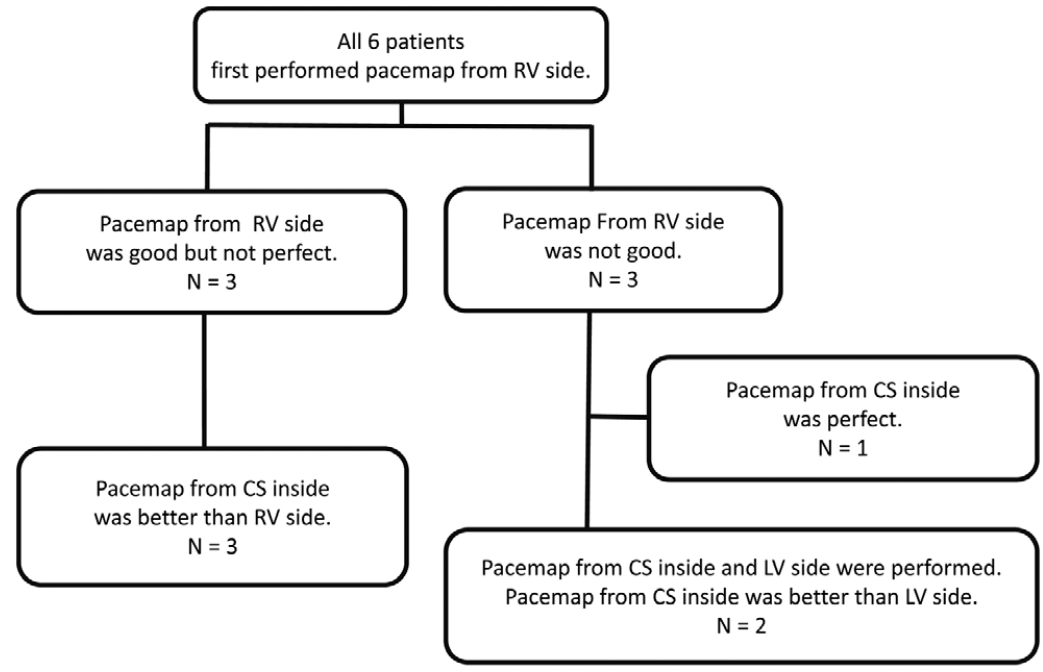

Figure 2. Mapping process in this study: 3 patients showed good but not perfect mapping from the right ventricular (RV) side and 3 cases did not show good pace mapping from the RV septum. Of these 3 patients, 1 patient showed perfect pace mapping from inside the coronary sinus (CS). The other 2 patients showed good mapping from the LV side, but pace mapping from inside the CS was better.

\section{Table 2. Electrophysiological Findings and Follow-up of the 6 Study Patients}

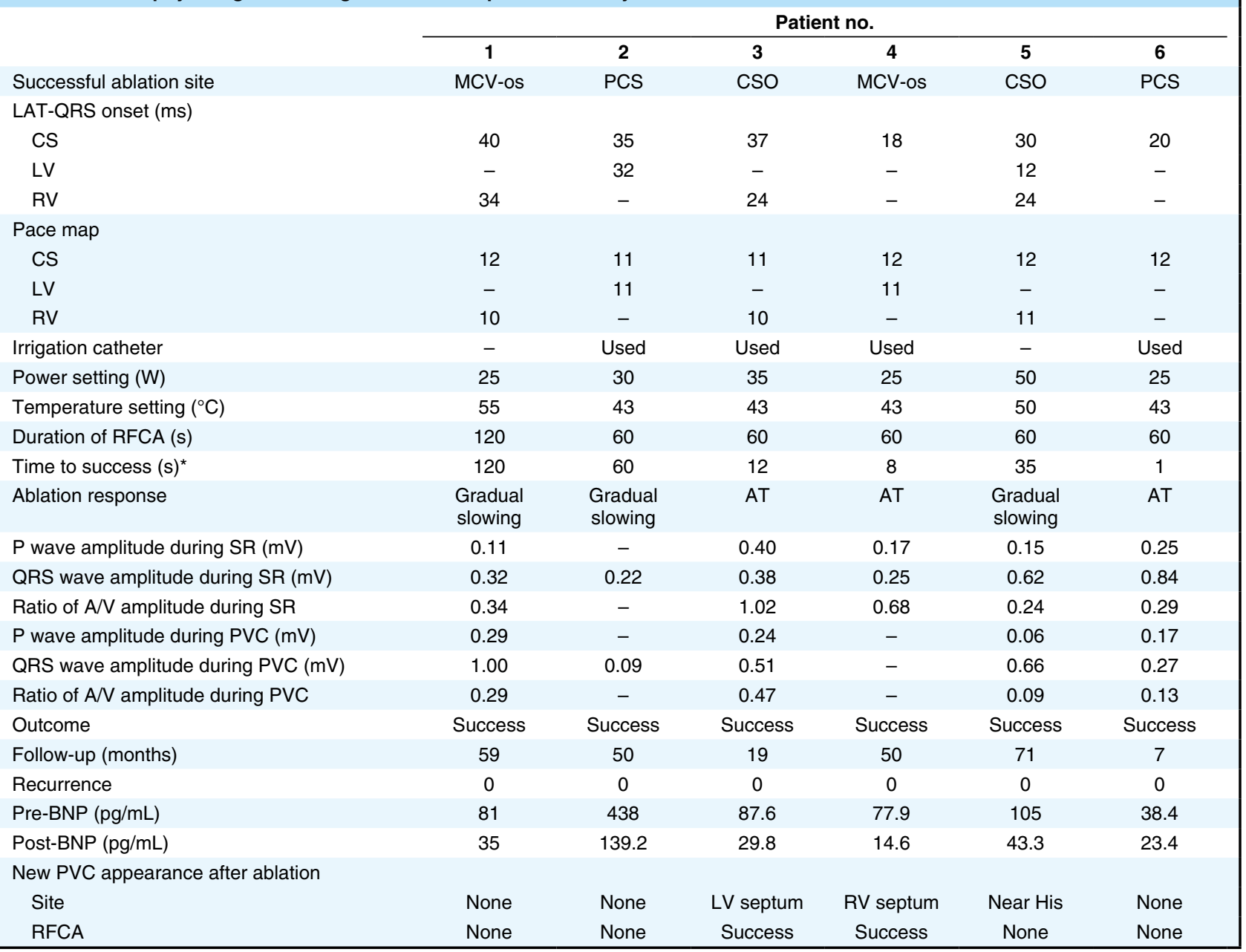

*Time from starting RFCA to the disappearance of ventricular arrhythmias. AT, abrupt termination; A/V ratio, atrial (P wave)/ventricular (QRS wave) ratio; LAT, local activation time; LV, left ventricle; pre/post BNP, serum B-type natriuretic peptide level pre/post RFCA; RFCA, radiofrequency catheter ablation; RV, right ventricle; SR, sinus rhythm. Other abbreviations as in Table 1. 

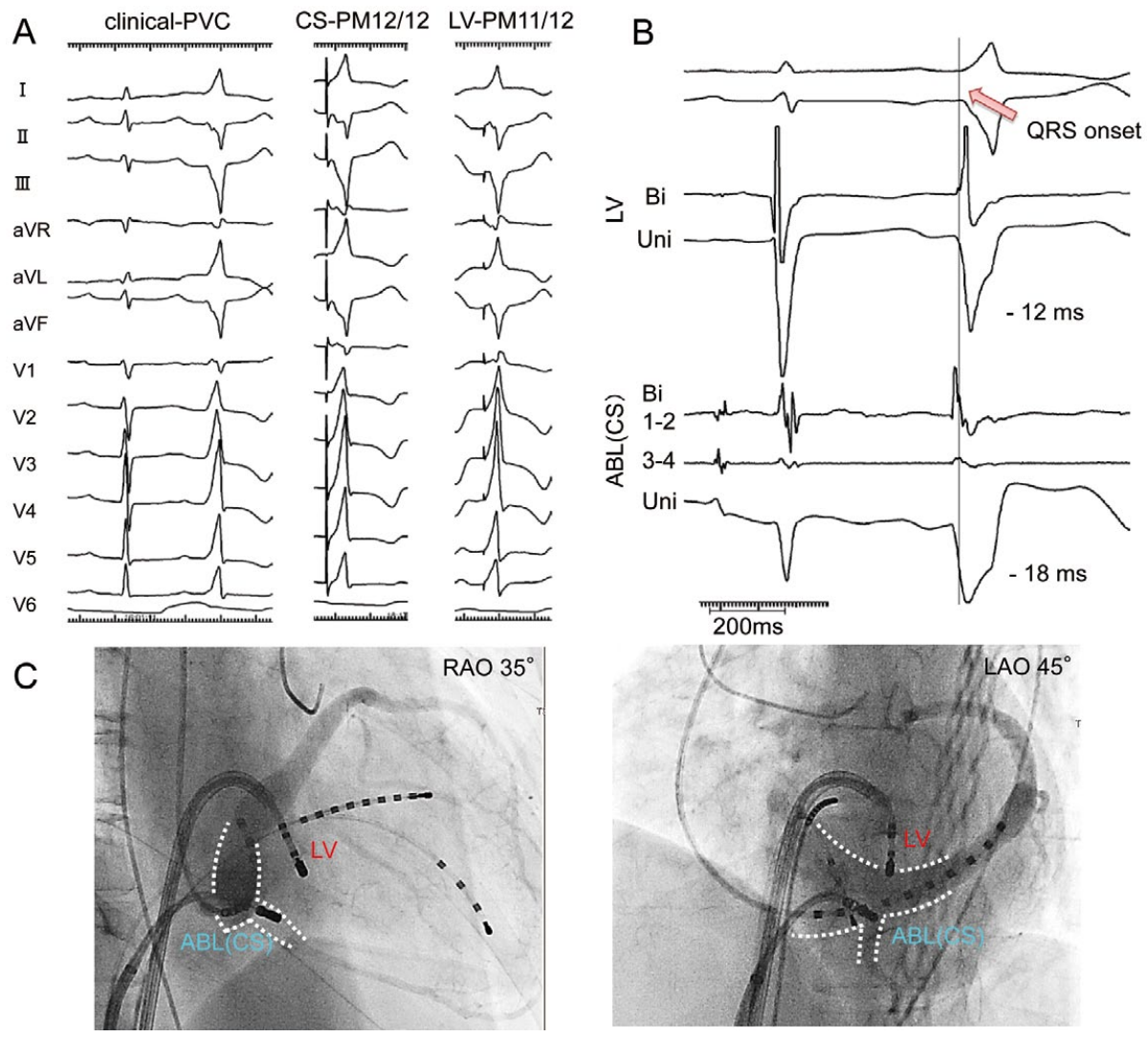

Figure 3. (A) Example of premature ventricular contraction (PVC) originating from the ostium of the middle cardiac vein. The pace map at the coronary sinus (CS-PM) is perfect (12/12), but the pace map at the posteroseptal LV (LV-PM) is good but not perfect (11/12). (B) Intracardiac ECG from an ablation catheter inside the coronary sinus ostium (CSO) at the successful ablation site, and from the left side of the posteroseptum. The ablation catheter inside the CSO is shorter than the left ventricular (LV) catheter through the atrial septum. The atrial/ventricular (A/N) ratio at the distal part of the ablation catheter (Abl) is $0.68(0.17 / 0.25 \mathrm{mV})$. (C) Fluoroscopic images of the Abl's position in the ostium of the middle cardiac vein. $\mathrm{ABL}(\mathrm{CS})$, ablation catheter inside coronary sinus; $\mathrm{Bi}$, bipolar electrogram; LAO, left anterior oblique; LV, left ventricle; RAO, right anterior oblique; Uni, unipolar electrogram.

branch block pattern. Only patient 4 showed a small "s" wave in leads V4-6.

\section{Characteristics of the Epicardial Origin of VAs}

The precordial MDI was $\geq 0.55(0.62 \pm 0.05)$ in all patients (Table 1). No patients showed a pseudo-delta wave duration $\geq 34 \mathrm{~ms}$ (mean: $23.2 \pm 5.3 \mathrm{~ms}$ ), and only 1 (17\%) patient showed an intrinsicoid deflection time in lead V2 $>85 \mathrm{~ms}$ (mean: $73.3 \pm 13.5 \mathrm{~ms}$ ). The criteria for the shortest RS complex duration matched our data in 4 of 5 patients (mean: $130.3 \pm 19.5 \mathrm{~ms})$.

\section{Results of Mapping and Ablation}

In all 6 patients, target VAs occurred spontaneously or with isoproterenol infusion. VAs could not be induced by ventricular-programmed stimulation and did not show entrainment or the resetting phenomenon during tachycardia, which clarified that the mechanism of these VAs might be abnormal automaticity. Figure 2 shows the mapping process of this study. Of the 6 patients, 3 showed good, but not perfect, mapping from the RV side. These 3 patients showed better pace mapping from inside the CS than by the RV septum. The other 3 did not show good pace mapping from the RV septum: 1 patient showed perfect pace mapping from inside the CS, so ablation delivery was performed from inside the CS; the other 2 patients showed good mapping from the LV side, but pace mapping from inside the CS was better than from inside the LV. Local activation time from the catheter located near the CSO to the onset of the QRS morphology was $30.0 \pm 9.1 \mathrm{~ms}$. In all 6 cases, the local activation time from the CSO was less than that from the LV $(22.0 \pm 14.1 \mathrm{~ms}$, range: $12-32 \mathrm{~ms}$ $[\mathrm{n}=2])$ and $\mathrm{RV}(27.3 \pm 5.7 \mathrm{~ms}$, range: $24-34 \mathrm{~ms}[\mathrm{n}=3])$. The pace map score at the CSO was relatively better (11-12 of 12) than that at the LV or RV side in each patient. All 6 patients were treated with RFCA from the CSO. The duration from RF energy delivery to elimination of VAs ranged from 1 to $120 \mathrm{~s}$ at the CSO.

\section{Electrophysiological Characteristics of VAs}

Intracardiac ECGs of successful ablation sites were precisely examined during sinus rhythm and clinical VAs, except in the case of 1 patient who had atrial fibrillation. During sinus rhythm, the mean atrial and ventricular wave amplitudes were $0.21 \pm 0.11 \mathrm{mV}$ and $0.43 \pm 0.24 \mathrm{mV}$, respectively, and the atrial/ventricular $(\mathrm{A} / \mathrm{V})$ amplitude ratio was $0.51 \pm 0.33$ (Table 2). During VAs, in patient number 4 (Table 2), the atrial wave and ventricular wave completely 


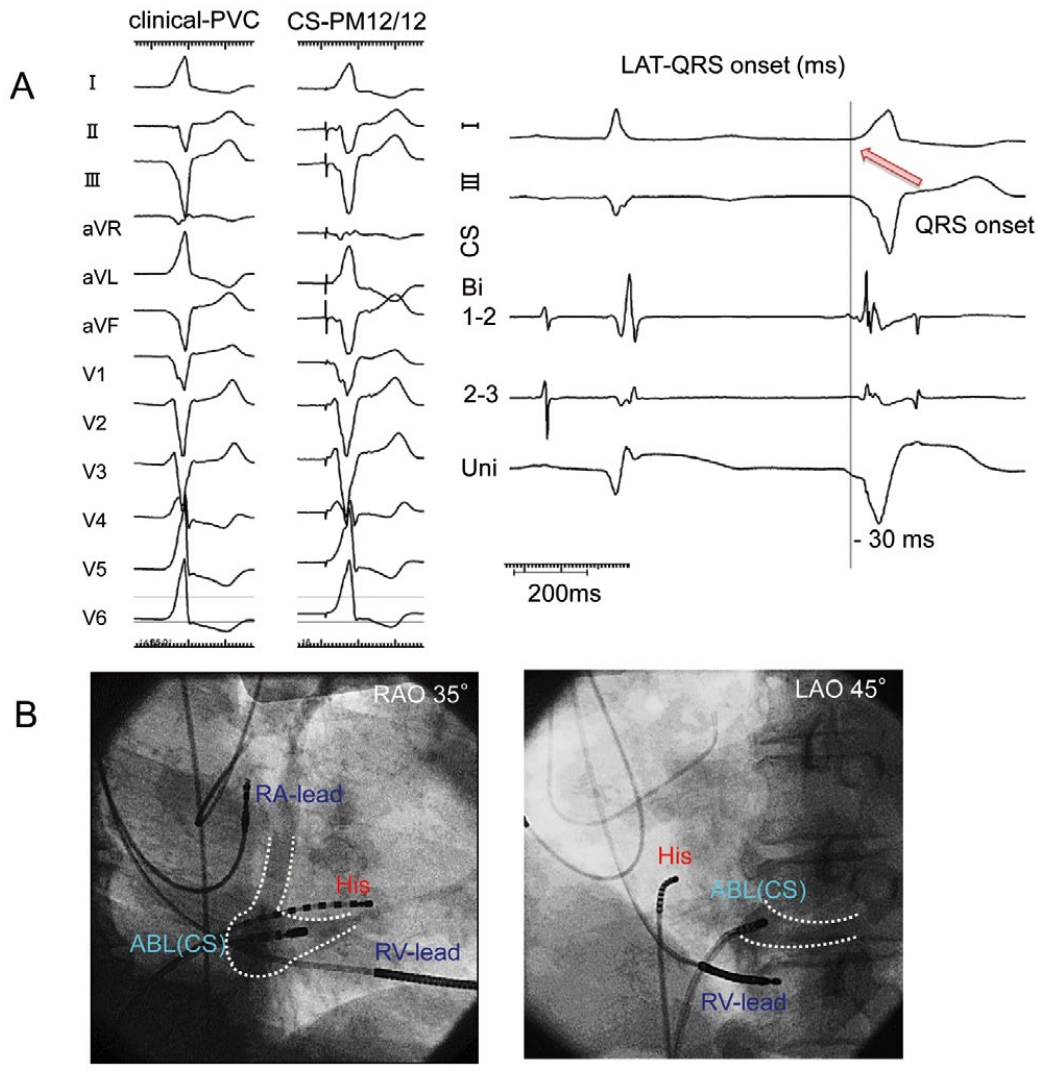

Figure 4. (A) Pace map and intracardiac electrocardiogram from a case of PVC of the CSO. The PVC shows a left bundle branch block pattern, and a perfect pace map at the CS is shown (CS-PM=12/12). The intracardiac ECG shows that the ablation site success precedes QRS onset for $30 \mathrm{~ms}$. The AN ratio at the distal part of the ablation catheter is 0.24 $(0.15 / 0.62 \mathrm{mV})$. (B) Fluoroscopic images of the position of the ablation catheter in the CSO. LAT, local activation time; RA, right atrium; RV, right ventricle. Other abbreviations as in Figures 2,3.

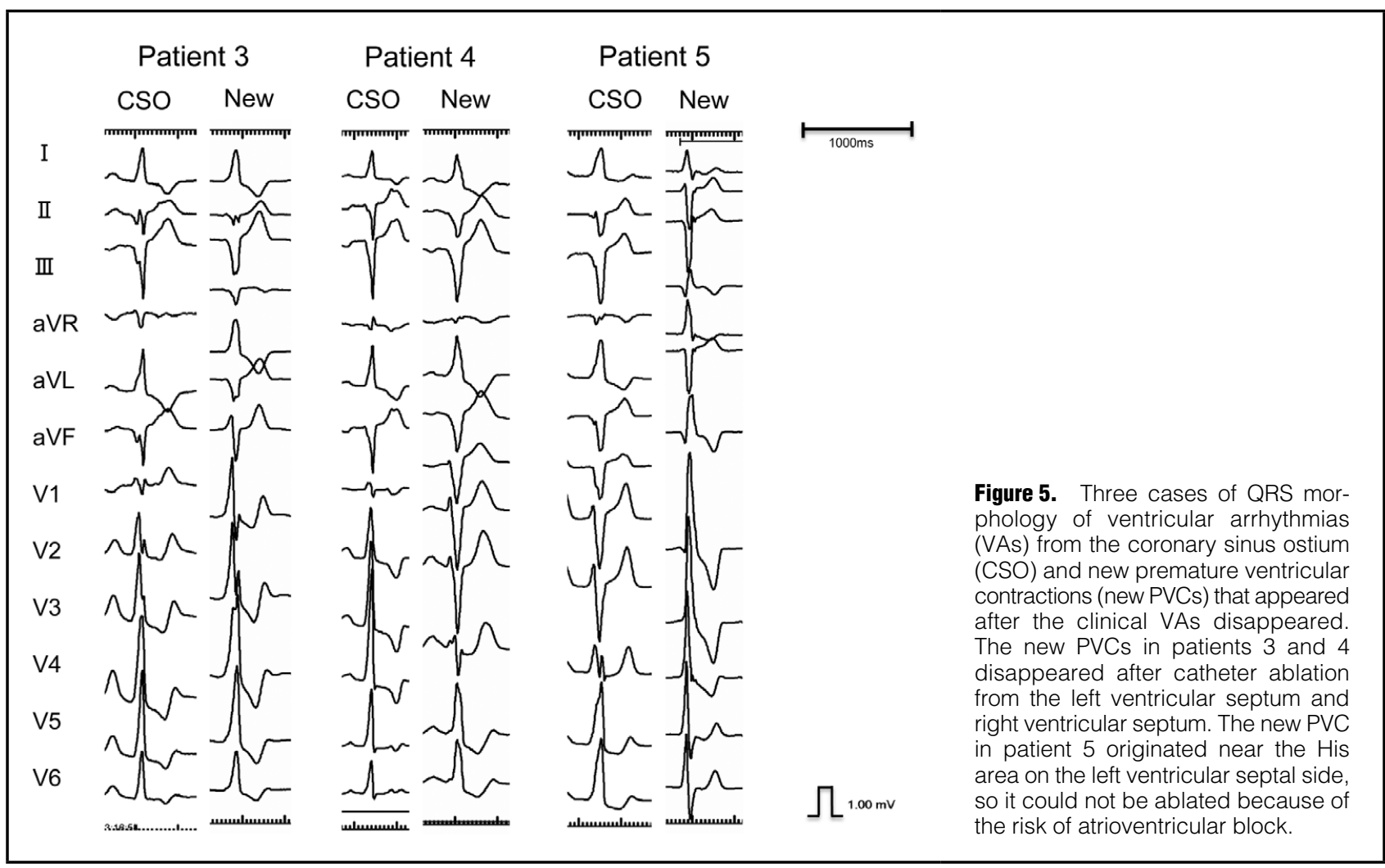




\begin{tabular}{|c|c|c|c|}
\hline & Our study & $\begin{array}{l}\text { Doppalapudi } \\
\text { et al }\left.\right|^{15}\end{array}$ & $\begin{array}{c}\text { Kawamura } \\
\text { et al }{ }^{18}\end{array}$ \\
\hline $\mathrm{n}$ & 6 & 4 & 9 \\
\hline Age (years) & $57-77$ & $31-79$ & $55-63$ \\
\hline Male & $3 / 6$ & $3 / 4$ & $3 / 9$ \\
\hline LVEF (\%) & $50-68$ & $45-55 \sim$ & $55-63$ \\
\hline \multicolumn{4}{|l|}{ VA pattern } \\
\hline PVC & $3 / 6$ & $0 / 4$ & $2 / 9$ \\
\hline SVT & $1 / 6$ & $4 / 4$ & $7 / 9$ \\
\hline QRS duration (ms) & $130-170$ & $150-165$ & $108-210$ \\
\hline MDI & $0.56-0.70$ & $0.58-0.64$ & $0.56-0.71$ \\
\hline \multicolumn{4}{|l|}{ VA morphology } \\
\hline LBBB type & $1 / 6$ & $3 / 4$ & $9 / 9$ \\
\hline RBBB type & $5 / 6$ & $1 / 4$ & $0 / 9$ \\
\hline \multicolumn{4}{|l|}{ VA axis } \\
\hline Left superior & $6 / 6$ & $4 / 4$ & $9 / 9$ \\
\hline Activation time from the CS (ms) & $18-40$ & 20-35 & 20-35 \\
\hline Successful ablation from the CS & $6 / 6$ & $1 / 4$ & $9 / 9$ \\
\hline Successful epicardial ablation & - & $2 / 4$ & - \\
\hline Complications & $0 / 6$ & $0 / 4$ & $0 / 9$ \\
\hline
\end{tabular}

CS, coronary sinus; LBBB, left bundle branch block; RBBB, right bundle branch block; VA, ventricular arrhythmia. Other abbreviations as in Table 1.

fused, so the amplitudes could not be measured exactly. In the other 4 patients, the mean atrial wave amplitude, ventricular wave amplitude, and $\mathrm{A} / \mathrm{V}$ amplitude ratio were $0.19 \pm 0.09 \mathrm{mV}, 0.50 \pm 0.35 \mathrm{mV}$, and $0.24 \pm 0.17$, respectively. In all cases, pace mapping was performed at the CSO. In 4 of 5 cases, 2 different pace maps were obtained at the same site, which were either atrial capture only or both ventricular and atrial capture. The local bipolar potentials from the successful ablation sites where the catheter was located were preceded by $10.0 \pm 3.6 \mathrm{~ms}$, which was earlier than the local unipolar potentials. In all patients, unipolar ECGs of successful ablation sites showed a "QS" pattern. Two examples of VAs originating from the CSO are shown in Figure 3 and Figure 4.

\section{Changed or New Morphology of VA After Elimination of Clinical VA}

In 3 patients, new VAs appeared after elimination of the clinical VAs (Table 2, Figure 5). The new VAs originated from the LV septum, the RV septum, and near the His area. These new VAs were not observed clinically before catheter ablation. Two VAs completely resolved with RFCA, while the third, which originated near the His area, did not resolve with RFCA.

\section{Acute Outcome}

In 1 patient, occasional clinical VAs persisted until the end of the session. However, the frequency of clinical premature ventricular contractions decreased. In addition, VAs disappeared several hours after RFCA, which was considered as delayed success. In the other 5 patients, VAs did not recur after ablation. There were no major complications related to the ablation procedure, such as cardiac tamponade, steam pop, or heart block. Moreover, we observed no changes in the ST segment and T wave on the 12-lead ECG or development of symptoms, including chest pain, after catheter ablation, which suggested there was no injury to the coronary arteries during the procedure.

\section{Follow-up and Midterm Outcome}

The mean follow-up period was $3.4 \pm 1.8$ years (range: $7-71$ months). Clinical VAs originating from the CSO did not appear in any 12-lead ECG or 24-h Holter monitoring. The symptoms related to VA resolved in all patients after RFCA. Serum B-type natriuretic peptide levels significantly decreased after the ablation procedure (before RFCA: $137.9 \pm 148.6 \mathrm{pg} / \mathrm{mL} ;$ after RFCA: $47.5 \pm 45.9 \mathrm{pg} / \mathrm{mL} ; \mathrm{P}=0.027)$.

\section{Discussion}

\section{ECGs of VAs Successfully Ablated From the CSO}

The VAs successfully ablated from the CSO had similar, but different ECG patterns, compared with those for other idiopathic VAs. VAs successfully ablated from the posteroseptum of the mitral annulus often exhibit an $\mathrm{rS}$ rather than a QS pattern in the inferior leads and taller $\mathrm{R}$ waves in V1. ${ }^{3}$ Though VAs originating from the posteroseptum of the tricuspid annulus and VAs successfully ablated from the CSO are frequently recorded as left bundle block patterns, the MDI may be a useful tool to distinguish between these. ${ }^{4}$ Moreover, VAs originating from both the posterior papillary muscle and left posterior fascicle have taller $\mathrm{R}$ waves in the V1 lead and deeper S waves in the V5 and V6 leads. ${ }^{5}$

In the present study patients, both atrial and ventricular potentials on intracardiac ECGs could be recognized from the catheters locating at the successfull ablation points in all cases. In previous reports, ${ }^{15,18}$ intracardiac ECGs of patients with VAs successfully ablated from the CSO were mentioned less frequently. The ratio of $\mathrm{A} / \mathrm{V}$ amplitude has great variability between patients. However, the mean A/V amplitude ratio recording from successful cases of catheter ablation in this study was $0.48 \pm 0.31$, suggesting that the VAs originated from the basal ventricle close to the tricuspid 
or mitral annulus. Based on our results, it might be important to search for the site at which both atrial and ventricular potentials can be equally recorded from the ablation catheter when attempting RFCA. Moreover, electrical pacing from the successful ablation sites captured both ventricular and atrial potentials in 4 of 5 patients. When the pace map from the CSO can be obtained by atrial capture, high-output pacing is strongly recommended at the same site before the ablation procedure.

\section{Comparison With Previous Studies on Crux VAs}

A comparison of previous studies on crux VAs is shown in Table 3. Doppalapudi et al reported idiopathic VTs originating from the epicardium at the crux. ${ }^{15}$ Kawamura et al also identified VAs from the area of the CSO as basal crux VAs. ${ }^{18}$ Although most crux VA patients showed left bundle branch QRS morphology, ${ }^{15,18}$ in our study 1 patient showed left bundle branch morphology and the other 5 patients exhibited right bundle branch morphology. The QRS morphology of VAs depends on the exit site. Generally, the CSO opens to the right atrial posterior septum and goes through the epicardial side of the LV. However, there were few reports of the association between the CSO and ventricular muscles. ${ }^{19,20}$ It might be difficult to judge whether ventricular muscle from the epicardium through the CSO is more related to the right or left ventricle. In 3 of the study patients, new VAs appeared just after the elimination of the clinical VAs. These new VAs were not observed prior to catheter ablation, which meant that they were most likely caused by exit changes in the clinical VAs from the extensive ablation. Kawamura et al reported that a spontaneous change from left bundle branch type to right bundle branch type in VT occurred in their apical crux group. ${ }^{18}$ This indicated a change in the exit site of the VAs. For VAs originating closer to the ventricular septum, depending if they are near the right or left side, the QRS morphology may change drastically. In addition, regarding the criteria for epicardial origin of the VA, a precordial MDI $\geq 0.55$ was found in all patients. However, no patient showed pseudo-delta wave duration $\geq 34 \mathrm{~ms}$ in this study. This result is in contrast with the findings of Kawamura et al, ${ }^{18}$ who showed that in $94 \%$ of their cases the patient had a pseudo-delta wave duration $\geq 34 \mathrm{~ms}$. Our interpretation is that the VAs originating from the CSO exited not only from the epicardial side, but also the endocardial side. Furthermore, 3 cases in this study exhibited changes in the VA exit site after treatment with RF energy, and 2 were treated from the endocardial side. This suggested that the origin did not exist completely on the epicardial side of the myocardium. There is no distinction between the right and left ventricle on the epicardial side of the myocardium, and myocardial fibers run in the transverse direction. Therefore, VAs may easily change their exit site to the right or left side, rather than the superior or inferior side.

\section{Complications Following RFCA at the CSO}

There are several important points to avoid serious complications associated with RFCA at the CSO. First, there may be a risk of occlusion or stenosis of the coronary artery with RF energy delivered to the inside of the CS. The CSO is located near the distal part of the left circumferential artery, right coronary artery, and atrial node artery. It is important to perform left and right coronary angiography to confirm the location of the ablation catheter, and not to perform RFCA when the distance between the ablation catheter and coronary artery is within $5 \mathrm{~mm}$. Second, the effect on the esophagus, which is near the posterior wall of the heart, should be considered. In atrial fibrillation ablation, a case of esophageal injury by catheter ablation from the inside of the CS has been reported. ${ }^{21}$ It would be beneficial to identify the location and shape of the esophagus with contrast studies to avoid serious complications. Third, we should pay attention to the occurrence of atrioventricular blocks caused by RFCA at the ostium of the CS. This area is adjacent to the slow pathway zone on the RV side. Therefore, it is important to note that RF applications may affect atrioventricular nodal conduction. Some atypical cases of atrioventricular nodal reentrant tachycardia have been successfully treated by catheter ablation from inside the CS. ${ }^{22}$ Finally, cardiac tamponade caused by catheter manipulation within the CS might become more severe. The use of a contact force catheter may be safer, as it can avoid excessive catheter contact within the CS. ${ }^{23}$

\section{Study Limitations}

First, only 6 cases were included. Considerably more cases are required to determine the true morbidity of these arrhythmias. Moreover, in past failure cases, VAs originating from the CSO may be included. Furthermore, the usual percutaneous epicardial approach was not used in this study, and we did not perform mapping from the LV side in all cases. In addition, an irrigation catheter was not used in all cases because of variable insurance coverage. Santangeli et al reported that VAs arising from the left posterior-superior process of the LV can be successfully eliminated from the adjacent right atrium. ${ }^{24}$ Although these VAs seemed to be similar to those in our report, we did not have any electrophysiological mapping data from the right atrium. Finally, understanding the relationship between the anatomy of the CS and the arrhythmogenic origin was important to mapping. Recently, intracardiac echocardiography has appeared to be useful for confirming the position of mapping catheters more accurately. In this retrospective study, the actual catheter position was not confirmed by intracardiac echocardiography imaging, which would have been helpful. Instead, CS venography was performed to confirm that the ablation catheter was located inside the CSO. It is possible that if we had used these other approaches or tools, RFCA would succeed at sites other than those reported herein or at sites that are suitable for ablation, as defined in this study.

\section{Conclusions}

Idiopathic VAs rarely arise from the epicardium at the CSO. In the present study, such VAs were eliminated by RFCA within the CS in cases where a clear atrial potential could be recorded.

\section{Disclosures}

Y.S. belongs to a department endowed by St. Jude Medical, and A.N belongs to a department endowed by Johnson \& Johnson and Medtronic. The authors received no grants or funding related to this research.

\section{References}

1. Komatsu Y, Taniguchi H, Miyazaki S, Kusa S, Takayama K, Kakita K, et al. Two distinct electrocardiographic forms of idiopathic ventricular arrhythmia originating in the vicinity of the His bundle. Europace 2012; 14: 1778-1785. 
2. Nogami A, Naito S, Tada H, Taniguchi K, Okamoto Y, Nishimura S, et al. Demonstration of diastolic and presystolic Purkinje potentials as critical potentials in a macroreentry circuit of verapamil-sensitive idiopathic left ventricular tachycardia. $J$ Am Coll Cardiol 2000; 36: 811-823.

3. Tada H, Ito S, Naito S, Kurosaki K, Kubota S, Sugiyasu A, et al. Idiopathic ventricular arrhythmia arising from the mitral annulus. J Am Coll Cardiol 2005; 45: 877-886.

4. Tada H, Tadokoro K, Ito S, Naito S, Hashimoto T, Kaseno K, et al. Idiopathic ventricular arrhythmias originating from the tricuspid annulus. Heart Rhythm 2007; 4: 7-16.

5. Yamada T, Doppalapudi H, McElderry HT, Okada T, Murakami Y, Inden Y, et al. Electrocardiographic and electrophysiological characteristics in idiopathic ventricular arrhythmias originating from the papillary muscles in the left ventricle. Circ Arrhythm Electrophysiol 2010; 3: 324-331.

6. Crawford T, Mueller G, Good E, Jongnarangsin K, Chugh A, Pelosi F Jr, et al. Ventricular arrhythmias originating from papillary muscles in the right ventricle. Heart Rhythm 2010; 7: $725-730$.

7. Tada H, Nogami A, Naito S, Fukazawa H, Horie Y, Kubota S, et al. Left ventricular epicardial outflow tract tachycardia: A new distinct subgroup of outflow tract tachycardia. Jpn Circ J 2001; 65: $723-730$

8. Yamada T, McElderry HT, Doppalapudi H, Okada T, Murakami Y, Yoshida Y, et al. Idiopathic ventricular arrhythmias originating from the left ventricular summit. Circ Arrhythm Electrophysiol 2010; 3: 616-623.

9. Daniels DV, Lu YY, Morton JB, Santucci PA, Akar JG, Green A, et al. Idiopathic epicardial left ventricular tachycardia originating remote from the sinus of Valsalva. Circulation 2006; 113: $1659-1966$

10. Berruezo A, Mont L, Nava S, Chueca E, Bartholomay E, Brugada J. Electrocardiographic recognition of the epicardial origin of ventricular tachycardias. Circulation 2004; 109: 1842 1847.

11. Obel OA, d'Avila A, Neuzil P, Saad EB, Ruskin JN, Reddy VY. Ablation of left ventricular epicardial outflow tract tachycardia from the distal great cardiac vein. J Am Coll Cardiol 2006; 48: $1813-1817$.

12. Kaseno K, Tada H, Tanaka S, Goto K, Yokokawa M, Hiramatsu S, et al. Successful catheter ablation of left ventricular epicardial tachycardia originating from the great cardiac vein. Circ J 2007; 71: 1983-1988.

13. Hirasawa Y, Miyauchi Y, Iwasaki YK, Kobayashi Y. Successful radiofrequency catheter ablation of epicardial left ventricular outflow tract tachycardia from the anterior interventricular coronary vein. J Cardiovasc Electrophysiol 2005; 16: 1378-1380.

14. Meininger GR, Berger RD. Idiopathic ventricular tachycardia originating in the great cardiac vein. Heart Rhythm 2006; 3: 464-466.

15. Doppalapudi H, Yamada T, Ramaswamy K, Ahn J, Kay GN. Idiopathic focal epicardial ventricular tachycardia originating from the crux of the heart. Heart Rhythm 2009; 6: 44-50.

16. Baman TS, Ilg KJ, Gupta SK, Good E, Chugh A, Jongnarangsin $\mathrm{K}$, et al. Mapping and ablation of epicardial idiopathic ventricular arrhythmias from within the coronaryvenous system. Circ Arrhythm Electrophysiol 2010; 3: 274-279.

17. Mountantonakis SE, Frankel DS, Tschabrunn CM, Hutchinson MD, Riley MP, Lin D, et al. Ventricular arrhythmias from the coronary venous system: Prevalence, mapping, and ablation. Heart Rhythm 2015; 12: 1145-1153.

18. Kawamura M, Gerstenfeld EP, Vedantham V, Rodrigues DM, Burkhardt JD, Kobayashi Y, et al. Idiopathic ventricular arrhythmia originating from the cardiac crux or inferior septum. Circ Arrhythm Electrophysiol 2014; 7: 1152-1158.

19. Inoue S, Becker AE. Koch's triangle sized up: Anatomical landmarks in perspective of catheter ablation procedures. Pacing Clin Electrophysiol 1998; 21: 1553-1558.

20. Shinbane JS, Lesh MD, Stevenson WG, Klitzner TS, Natterson PD, Wiener I, et al. Anatomic and electrophysiologic relation between the coronary sinus and mitral annulus: Implications for ablation of left-sided accessory pathways. Am Heart J 1998; 1: 93-98.

21. Güneş MF, Gökoğlan Y, Di Biase L, Gianni C, Mohanty S, Horton R, et al. Ablating the posterior heart: Cardioesophageal fistula complicating radiofrequency ablation in the coronary sinus. J Cardiovasc Electrophysiol 2015; 26: 1376-1378.

22. Otomo K, Nagata Y, Uno K, Fujiwa H, Iesaka Y. Atypical atrioventricular nodal reentrant tachycardia with eccentric coronary sinus activation: Electrophysiological characteristics and essential effects of left-sided ablation inside the coronary sinus. Heart Rhythm 2007; 4: 421-432.

23. Kuck KH, Reddy VY, Schmidt B, Natale A, Neuzil P, Saoudi $\mathrm{N}$, et al. A novel radiofrequency ablation catheter using contact force sensing: Toccata study. Heart Rhythm 2012; 9: 18-23.

24. Santangeli P, Hutchinson MD, Supple GE, Callans DJ, Marchlinski FE, Garcia FC. Right atrial approach for ablation of ventricular arrhythmias arising from the left posterior-superior process of the left ventricle. Circ Arrhythm Electrophysiol 2016; 9: $\mathrm{e} 004048$. 